\title{
Flow and evolution of ice-sucrose crystal mushes
}

\author{
Andrew J. Gilbert* \\ Department of Earth Sciences, University of Cambridge, Cambridge, United Kingdom \\ Felix K. Oppong ${ }^{\dagger}$ and Robert S. Farr ${ }^{\ddagger}$ \\ Unilever R\&D Colworth Science Park, MK44 1LQ, Bedford, United Kingdom
}

(Received 4 November 2016; published 11 April 2017)

\begin{abstract}
We study the rheology of suspensions of ice crystals at moderate to high volume fractions in a sucrose solution in which they are partially soluble, a model system for a wide class of crystal mushes or slurries. Under step changes in shear rate, the viscosity changes to a relaxed value over several minutes, in a manner well fitted by a single exponential. The behavior of the relaxed viscosity is power-law shear thinning with shear rate, with an exponent of $-1.76 \pm 0.25$, so that shear stress falls with increasing shear rate. On longer time scales, the crystals ripen (leading to a falling viscosity) so that the mean radius increases with time to the power $0.14 \pm 0.07$. We speculate that this unusually small exponent is due to the interaction of classical ripening dynamics with abrasion or breakup under flow. We compare the rheological behavior to mechanistic models based on flow-induced aggregation and breakup of crystal clusters, finding that the exponents can be predicted from liquid phase sintering and breakup by brittle fracture.
\end{abstract}

DOI: 10.1103/PhysRevE.95.042606

\section{INTRODUCTION}

The flow of crystal suspensions in which the crystals are soluble in the liquid phase (termed hereafter "mushes") is important in both nature and engineering. Examples from earth and planetary sciences involving this class of material include magmatic emplacement, lava flows [1-3], the formation of sea ice [4], and cryogenic eruptions [5-7]. In an artificial setting, they can occur in frozen foods [8,9], slurry-ice refrigerant systems [10], metal casting [11,12], slurry explosives [13], solution mining [14], and evaporative mineral and sugar refinement.

Our aims in this paper are to understand the rheology of high-volume-fraction ice suspensions (in a sucrose solution), as a model system for mushes in general, and to develop a constitutive model to explain the rheology, based on the physics of the interacting crystals.

Mushes consist of a suspension of hard, partially soluble crystals in a carrier liquid (which we term the "serum phase"), the latter being usually Newtonian in rheology. However, additional complexity arises because the crystals have attractive or adhesive interactions resulting from van der Waals forces [15] but more characteristically from their tendency to undergo liquid-phase sintering into clusters when they touch [16]. The importance of such attractive interactions will depend on the size and solubility of the crystals and the diffusivity of molecules in the serum, so one can anticipate a spectrum of behaviors from approximately hard particle suspension rheology to cases where sintering dominates.

As well as aggregation and sintering, soluble crystals will undergo ripening [17-19], driven by the minimization of interfacial energy as the larger crystals grow at the expense of the smaller. The mean crystal size will therefore gradually increase throughout any experiment.

\footnotetext{
*ajg204@cam.ac.uk

†felix.oppong@unilever.com

${ }_{\ddagger}^{\ddagger}$ robert.farr@unilever.com
}

These phenomena lead to complex rheological properties: Mushes are typically shear thinning at low solid volume fraction $\phi$ and can develop a yield stress and exhibit pseudoplastic behavior $[6,9,20]$. The time-dependent nature of cluster formation and of sintering can also lead to strong history dependence in the rheology. For example, if left unsheared, a mush will often solidify [9] and in general one would expect it to display thixotropic behavior [21] under changes in shear rate [6].

The flow of a suspension of hard particles with no interactions other than hydrodynamics was first studied by Einstein [22], who showed that spheres at a volume fraction $\phi \ll 1$ enhance the viscosity by a factor $(1+B \phi)$, where $B$ is a pure number, termed the "intrinsic viscosity" or Einstein coefficient. For spheres this is 2.5, but it is larger for other shapes [23]. At higher volume fractions (particularly above $\phi=0.2$ ), the viscosity increases more strongly than the Einstein result [24] and will in general diverge at a maximum volume fraction $\phi_{m}$, which may be related to random close [25] or loose [26] packing, or result from a dynamic process of dilatancy [27] or jamming [28]. For the volume fractions of interest in this paper, a widely used approximation for the suspension viscosity $\eta$ in terms of the continuous phase viscosity $\eta_{0}$ is from Krieger and Dougherty [29]:

$$
\eta=\eta_{0}\left(1-\frac{\phi}{\phi_{m}}\right)^{-B \phi_{m}},
$$

where for subspherical particles of roughly equal size, one would use $B=2.5$ and $\phi_{m} \approx 0.64$. Lower values of $\phi_{m}$ might be due to frictional interactions, leading to divergence at loose packing or lower packing density due to significant departure from sphericity [30,31]. Higher values may arise from polydispersity $[32,33]$.

When attractive forces between particles are present, shear thinning and other non-Newtonian behavior can result. For very dilute suspensions, aggregation leads to fractal flocs $[34,35]$, which can ultimately percolate to form a gel. Before gelation, such suspensions are weakly shear thinning, 
while afterward the gels are viscoelastic solids [36] with properties that scale with $\phi$. At higher volume fractions (the subject of this paper), there is no universal theory of attractive particle rheology and flow properties depend on the details of the interparticle interactions. Strongly cohesive suspensions can display steep shear thinning and nonmonotonic flow curves [37,38].

When making rheological measurements on such systems, pseudoplasticity introduces problems in simple geometries. Using a parallel plate or Couette flow cell will lead to flow instabilities such as shear banding [39], while capillary rheometers may be subject to plug flow [40]. These phenomena leave the majority of the volume undeformed, so one is only probing the flow in thin layers of the fluid. Moreover, with a history-dependent mush, the unsheared regions are likely to solidify, exacerbating the problem. In this paper, we therefore use a more complex geometry to ensure bulk deformation of the material. This consists of a cylindrical vessel with a rotating impeller for which we measure both the rotation rate $\omega$ and the applied torque $T$.

A second problem arises from the particle size of the suspension: For reliable measurements, the flow geometry should ensure that the gaps through which the suspension is forced to flow are large compared to a crystal (or cluster), so that most of the viscous dissipation occurs in the bulk of the fluid rather than in locally jammed regions in confined spaces [41]. This, together with the size of the crystals and the values of the interesting shear rates, mean that we are not able to guarantee that the Reynolds number Re of the flow is small.

All these considerations mean that calibration of the rheological apparatus is nontrivial, and for our case will rely on some specific assumptions about the rheology, which we are then able to test post hoc.

\section{OUTLINE AND KEY ASSUMPTIONS}

Throughout this paper, we suppose that under constant rootmean-square shear rate $\dot{\gamma}_{\text {rms }}$, the viscosity has a power law dependence on this shear rate and the mean crystal radius $R$, with a volume-fraction-dependent prefactor:

$$
\eta \propto \dot{\gamma}_{\mathrm{rms}}^{n_{s}} R^{n_{r}} .
$$

Here, $n_{s}$ and $n_{r}$ are exponents which we obtain in Sec. VD. We hypothesize that this behavior arises from crystal clusters dynamically formed and broken up in the flow. At high shear rates, we anticipate that the clusters will be broken down, and there will be a crossover to Newtonian behavior with the Krieger-Dougherty value for the viscosity.

We also suppose that crystal ripening will occur and lead to a power-law dependence of crystal radius on time $t$ and potentially also on root-mean-square shear rate (again with a volume-fraction-dependent prefactor):

$$
R\left(\dot{\gamma}_{\mathrm{rms}}, t\right) \propto t^{p_{t}} \dot{\gamma}_{\mathrm{rms}}^{p_{s}},
$$

where $p_{t}$ and $p_{s}$ are exponents we find in Sec. V C.

Together, Eqs. (2) and (3) imply that the observed viscosity in experiments at constant volume fraction $\phi$, as a function of time and constant shear rate, will have the following form:

$$
\eta\left(\dot{\gamma}_{\mathrm{rms}}, t\right) \propto t^{n_{r} \cdot p_{t}} \dot{\gamma}_{\mathrm{rms}}^{n_{r} \cdot p_{s}+n_{s}} .
$$

These exponent combinations are determined in Secs. V A and VC. The value for $n_{s}$ can be obtained more easily from experiments where the shear rate is suddenly changed (see Sec. V B).

The viscometer employed was first calibrated over a range of Reynolds numbers, using Newtonian fluids and hard sphere suspensions of known viscosity, similar to the crystal mushes we are ultimately interested in. The calibration (Sec. IV) allows one to deduce viscosity from $T$ and $\omega$ (and thus power dissipation) and also to attribute to the flow root-mean-square values of shear rate $\dot{\gamma}_{\text {rms }}$ and shear stress $\tau_{\text {rms }}$.

In general, this calibration, performed for Newtonian fluids, cannot be used for non-Newtonian fluids. This is because the material, being subject to different shear rates at different locations, will also have a spatially dependent viscosity. The resulting flow pattern will not correspond to any of the velocity fields covered by the Newtonian calibration.

There is, however, a class of nontrivial rheologies to which the calibration does apply: Suppose that following a sudden change in shear rate, the viscosity is initially unchanged, but then relaxes thixotropically towards a new viscosity corresponding to the new shear rate. If this relaxation happens on a time scale that is long compared to a rotation time of the viscometer, then the viscosity will remain spatially uniform even as it changes slowly with time, and the Newtonian calibration can be used to deduce the viscosity even as it relaxes to the new steady-state value.

The hallmark of this behavior is that if the rotation rate is changed suddenly, the torque $T$ will change discontinuously, but the calculated viscosity should be essentially continuous (although its first derivative with time may be discontinuous). Furthermore, only a relatively small fraction of the ultimate change in viscosity should occur on a time scale of a single rotation of the impeller. As we see in Sec. V B, this is indeed observed for the crystal mushes studied here.

Microstructurally, we interpret this rheological behavior in the following way: If the dissipated power in the flow is coming primarily from the deformation of the Newtonian serum, then high values of crystal mush viscosity arise from the presence of crystal clusters [2], which are built up and break down dynamically in the flow. A sudden change in shear rate does not immediately affect the cluster statistics, so it only acts through a proportional change in the local flow rates in the serum phase, and hence gives an instantaneously Newtonian behavior of the suspension. Only over the course of several inverse shear rates does the aggregation and breakup dynamics converge to a different distribution of cluster sizes and shapes (and thus a relaxed viscosity). Although both aggregation and breakup are likely to occur more quickly at higher shear rates, it is to be expected that breakup will increase more quickly than aggregation, so that cluster size and hence viscosity will fall with increasing shear rate. The relaxed viscosity will therefore be shear thinning, a conclusion borne out by our results in Secs. V A and VB.

A further complication is that even at constant rotation rate, the viscosity falls gradually over time, which we interpret to be due to the slow growth by ripening of the crystals. Data on ripening are obtained by optical microscopy on samples taken from the suspension and presented in Sec. V C. 
Finally, in Sec. VI, we make simple theoretical predictions for the relaxed viscosity as a function of crystal size and suspension shear rate, based on theories of adhesive contact or sintering and fracture of crystal contacts, together with aggregation and breakup dynamics. To do this, we argue that at relatively high volume fractions and in the regime where viscosity is dominated by clustering, the root mean square shear stress $\tau_{\mathrm{rms}}$ in the suspension is

$$
\tau_{\mathrm{rms}} \propto \frac{F_{\max }}{R^{2}},
$$

where $F_{\max }$ is the force required to break an adhesive contact between two crystals, and the dimensionless prefactor depends only weakly on volume fraction.

This allows us to predict values for $n_{s}$ and $n_{r}$ in terms of theories for adhesion, liquid phase sintering, and fracture, and compare these to the experimentally measured values. The comparison of theory to experiment is shown in Fig. 16, and we find good agreement with a simple liquid phase sintering model and the evaporation-condensation theory of Kingery and Berg [42].

\section{MATERIALS AND METHODS}

The rheological apparatus used was a custom-made viscometer, developed by the engineering workshop at Unilever Research Colworth, and referred to hereafter as a "stirred pot" (Fig. 1). It consists of a jacketed cylindrical vessel of inner radius $R_{\text {pot }}=0.04 \mathrm{~m}$ and depth $H_{\text {pot }}=0.135 \mathrm{~m}$, maintained at a set temperature of $\Theta=-10^{\circ} \mathrm{C}$ by pumping propylene glycol solution through the jacket and a circulating, refrigerating Haake F8/C35 water bath. The impeller is an anchor geometry (see Fig. 1), which rotates at a chosen angular frequency $\omega$, while torque $T$ is recorded. We chose rotation rates in the range 200 to $400 \mathrm{rpm}\left(\omega=20.9\right.$ to $\left.41.9 \mathrm{rad} \mathrm{s}^{-1}\right)$. In experiments with

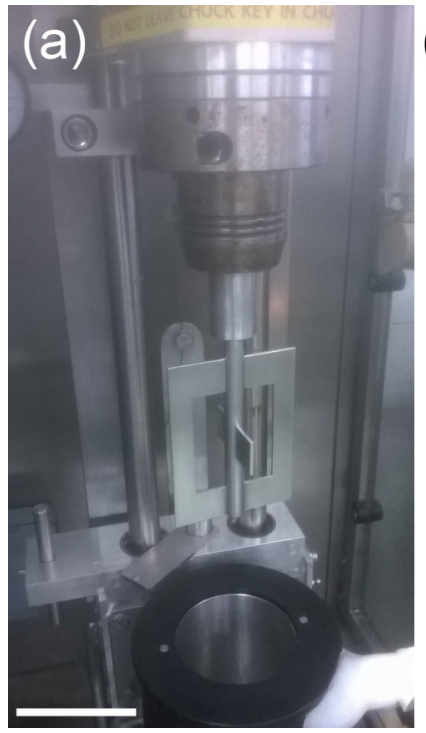

(b)

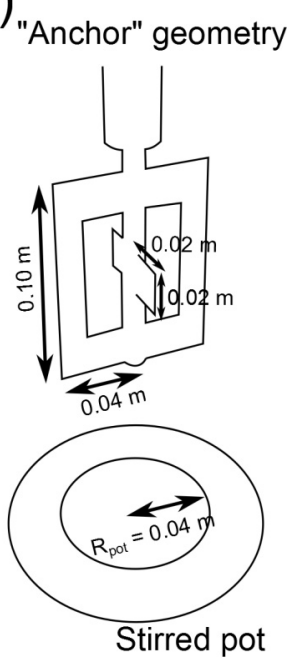

FIG. 1. (a) Image of "stirred pot" viscometer, with the anchor geometry impeller in the raised position. Scale bar is $10 \mathrm{~cm}$. (b) Line drawing of the anchor and stirred pot showing dimensions of the anchor paddles and stirred pot.
TABLE I. Formulations of ice-sucrose suspensions, along with the ice volume fraction expected for each suspension at $-10^{\circ} \mathrm{C}$.

\begin{tabular}{lcc}
\hline \hline Sucrose $($ wt $\%)$ & Water $($ wt $\%)$ & $\phi($ ice $)$ at $-10^{\circ} \mathrm{C}$ \\
\hline 55.0 & 45.0 & 0 \\
41.7 & 58.3 & 0.33 \\
37.1 & 62.9 & 0.42 \\
32.5 & 67.5 & 0.51 \\
28.8 & 70.2 & 0.57 \\
\hline \hline
\end{tabular}

no change in rotation rate, data are collected every $300 \mathrm{~s}$ over a run of several hours. In runs where we impose a step change in rotation rate, data were collected every $2 \mathrm{~s}$ to observe the transients arising from this change.

All experiments on ice-sucrose mushes were conducted at fixed temperature $\Theta=-10^{\circ} \mathrm{C} \pm 0.2^{\circ} \mathrm{C}$, so the ice volume fraction $\phi$ is determined by the sucrose concentration only, and the unfrozen serum phase has a fixed composition and therefore viscosity at this temperature. The sucrose concentrations used are shown in Table I, including a sucrose solution for which no crystals formed, hence for which $\phi=0$, which is precisely the composition of the serum phase in the ice-containing samples at this temperature. This zero-ice formulation, being Newtonian, was used for one of the calibration experiments. Sucrose solutions were prepared by adding boiled water to granulated sucrose and then cooling overnight to $+5^{\circ} \mathrm{C}$, before pouring into the stirred pot, which had been previously cooled to $-10^{\circ} \mathrm{C}$.

The remaining calibration experiments were conducted at a range of different $\Theta$ and used $90 \%$ and $99.5 \%$ glycerol ( $\rho=1260 \mathrm{~kg} \mathrm{~m}^{-3}$, from Sigma-Aldrich), either alone or as the serum phase in a suspension of silica spheres of $R=$ $100 \mu \mathrm{m}$ (with a range of $50150 \mu \mathrm{m}$ ) and density $1800 \mathrm{~kg} \mathrm{~m}^{-3}$ (see Fig. 2; also from Sigma-Aldrich). These suspensions are predicted to be Newtonian and behave according to the Krieger-Dougherty relation, Eq. (1). The viscosities of the glycerol without silica spheres, and the sucrose solution without ice, were measured using an Anton Paar MCR501 rheometer.

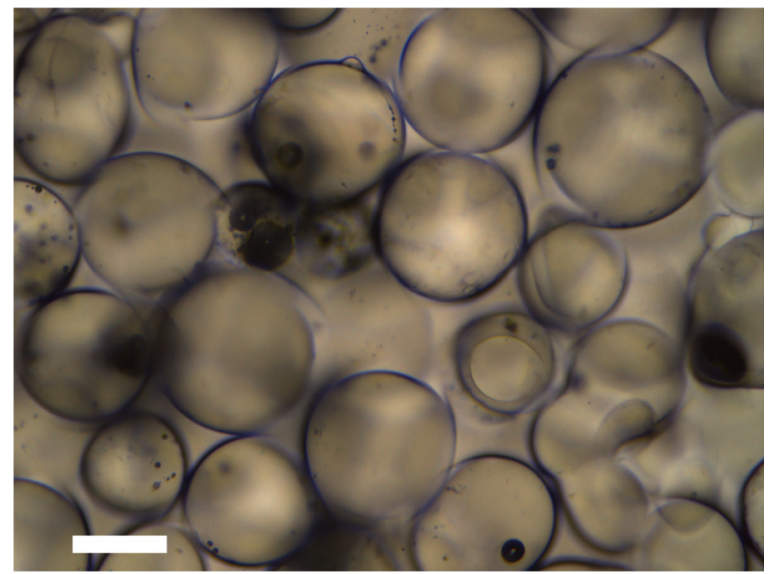

FIG. 2. Image of the silica spheres added to the glycerol. Scale bar $100 \mu \mathrm{m}$. 
In experiments where samples were extracted for image analysis, the stirrer was briefly stopped $(\approx 1 \mathrm{~min})$, a small portion of the sample was removed, and the temperature was checked. The torque was not affected by the stoppages and returned to its previous value after being switched back on. The images were produced using a Leica DMLM microscope and Leica DFC490 camera, and the slides were kept cool using a Linkam cold stage with the temperature regulated at $-10^{\circ} \mathrm{C}$ using a Landa RMB water bath. The crystals were then analyzed using Image J software to get values of crystal radius and aspect ratio. Two lines were drawn on an images of each crystals, one of maximum length and a second perpendicular to that. Using the ellipse function in ImageJ, the aspect ratio of the crystal can be measured. In order to measure crystal radius, the shorter line was removed and the perimeter function was used.

\section{CALIBRATION}

The aim of our calibration is to be able to deduce viscosity $\eta$ from torque $T$ and rotation rate $\omega$. In order to do this, we have performed a series of calibration experiments using Newtonian suspensions where viscosity is known, $\omega$ is set, and $T$ is measured. This allows us to come up with an equation to predict suspension viscosity from the measured parameters of the system.

First, we define a Reynolds number for our system as

$$
\operatorname{Re} \equiv \frac{\rho R_{\text {pot }}^{2} \omega}{\eta}
$$

where $\rho$ is the suspension density.

We cannot assume the experiments are performed at low Re, with a laminar flow, so the measurement equipment must be calibrated for a range of Reynolds numbers. In practice, for our experimental data set, we encounter a (calculated) range from $2<\operatorname{Re}<30$. More specifically, we use three rotation rates, $\omega=20.9,30.4$, and $41.9 \mathrm{~s}^{-1}$, and the Reynolds numbers found in each span the approximate ranges $2<\operatorname{Re}<5,10<\operatorname{Re}<$ 15 , and $20<\operatorname{Re}<30$ respectively. The calibration in this section covers the range $0.5<\operatorname{Re}<350$. The key assumption, when we later use this calibration to determine mush viscosity, is that this viscosity is spatially uniform in the stirred pot, even if the material is thixotropic and (on longer time scales) shear thinning. The experimental justification for this assumption is provided in Sec. VB.

The stirred pot was calibrated using $99.5 \%$ glycerol at different temperatures, either alone or as the liquid phase in a suspension of $100 \mu \mathrm{m}$ radii silica spheres at $+18,+20$ and $+25^{\circ} \mathrm{C}$ (see Fig. 2) and $90 \%$ glycerol at $+21^{\circ} \mathrm{C}$ with no spheres. A second calibration set was performed using $55 \%$ sucrose solution in water (with no silica spheres) at $-10^{\circ} \mathrm{C}$, a temperature and sucrose concentration where no ice is present (these systems are all Newtonian or predicted to be so from Krieger-Dougherty). A third calibration set was performed using golden syrup (Tate and Lyle Ltd) at $+25^{\circ} \mathrm{C}$; this set was performed to observe the limiting behavior at low Re. The viscosities of the glycerol and sucrose solutions were measured, while viscosities of the silica sphere suspensions were deduced from these values and the Krieger-Dougherty relation for spheres, Eq. (1), using $B=2.5$ and maximum packing fraction $\phi_{m}=0.64$. The viscosity of golden syrup is $45 \mathrm{~Pa} \mathrm{~s}$ at $+25^{\circ} \mathrm{C}[43]$.

The glycerol and silica sphere suspensions were placed in the stirred pot, and the torque $T$ and temperature $\Theta$ were recorded as a function of time $t$ at different angular velocities $\omega$. The silica sphere suspension volume fractions used were 0 , 0.42 , and 0.51 . For each run, measurements were taken over a time of $30 \mathrm{~min}$ and were seen to be steady during that time.

The purpose of the calibration is to allow us to deduce the viscosity of a fluid in the stirred pot from the torque and rotation rate. Consider therefore the time-average power $P$ dissipated by viscous flow in the stirred pot. Let $\langle\cdot\rangle$ denote an average over both space (within the pot) and time (over a few rotations), while $\dot{\gamma}$ is the local, instantaneous shear rate in the suspension. Then we note

$$
P=T \omega=V_{\text {pot }}\left\langle\eta \dot{\gamma}^{2}\right\rangle=V_{\text {pot }} \eta \dot{\gamma}_{\mathrm{rms}}^{2},
$$

where the volume occupied by the suspension is

$$
V_{\mathrm{pot}} \approx \pi R_{\mathrm{pot}}^{2} H_{\mathrm{pot}}
$$

and we have defined $\dot{\gamma}_{\text {rms }} \equiv\left\langle\dot{\gamma}^{2}\right\rangle^{1 / 2}$. We have also used the assumption that the viscosity is uniform and constant (over a few rotation times at least) to bring $\eta$ outside the spatiotemporal average.

For small $R e \ll 1$ (creeping flow) we would expect $\dot{\gamma}_{\text {rms }} \propto \omega$. However, for larger $R e \gg 1$, there may be a more complicated dependence. In general we take

$$
\dot{\gamma}_{\mathrm{rms}}=f(\mathrm{Re}) \omega
$$

for some function $f(R e)$ to be determined. The function $f$ represents is a correction to the low-Reynolds-number behavior, where it is a constant. For our system, $f$ does not vary by a large amount, and most of the change in $\dot{\gamma}_{\text {rms }}$ arises simply from the linear factor of $\omega$ in Eq. (9).

From Eqs. (7) and (9) we find

$$
f^{2}=\frac{T}{V_{\text {pot }} \omega \eta},
$$

so that Eqs. (6) and (10) allow us to plot $f^{2}$ as a function of $R e$ for the calibration experiments, as shown in Fig. 3.

At low $\mathrm{Re}, f^{2}$ must tend to a constant, while at higher Re, we find $f^{2} \propto \operatorname{Re}^{\frac{1}{2}}$ (approximately). We therefore fit $f^{2}$ to the following form:

$$
f^{2}=\left(C_{0}+C_{1} \operatorname{Re}\right)^{\frac{1}{2}} .
$$

Plotting up the data in Fig. 3, we find that a good fit can be obtained with $C_{0}=3$ and $C_{1}=0.66$. Rearranging and solving the quadratic equation allows us to deduce a (spatially uniform) viscosity for the later experiments from known and measured quantities of $T$ and $\omega$ :

$$
\eta \approx \frac{T \chi}{0.33 V_{\mathrm{pot}} \omega}\left[1+\left(1+\frac{3 \chi^{2}}{0.33^{2}}\right)^{\frac{1}{2}}\right]^{-1},
$$

where $\chi$ is a nondimensional quantity given as

$$
\chi \equiv \frac{T}{V_{\text {pot }} \rho \omega^{2} R_{\text {pot }}^{2}} .
$$




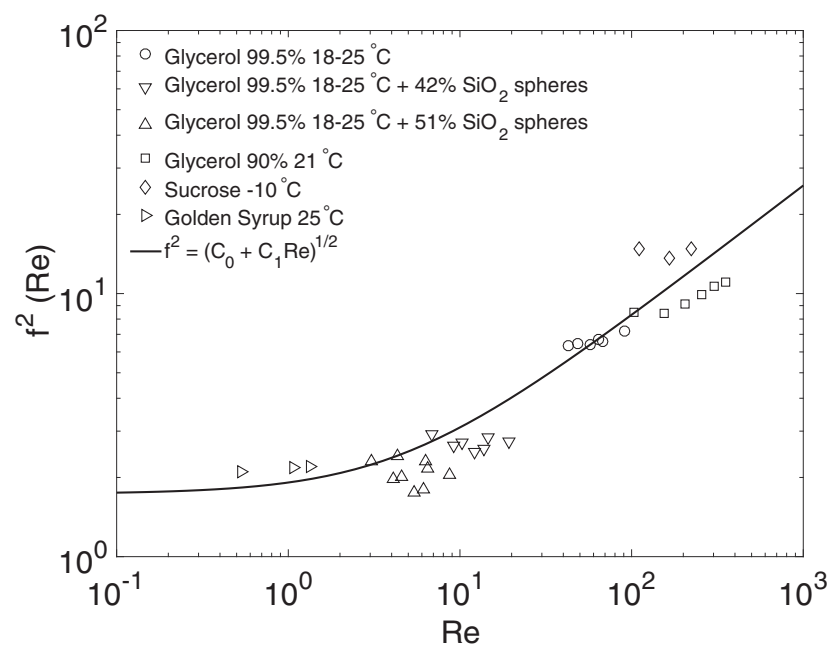

FIG. 3. Calibration curve showing how $f^{2}$ varies as a function of $\mathrm{Re}$ for the various calibration experiments that have been performed. The curve is from Eq. (11) using $C_{0}=3$ and $C_{1}=0.66$. This spans the values of Reynolds numbers from our experiments, which are calculated as $2<\operatorname{Re}<30$.

We will also want to calculate values for $\dot{\gamma}_{\text {rms }}$, which from Eq. (7) can be obtained from

$$
\dot{\gamma}_{\mathrm{rms}}=\left(\frac{T \omega}{V_{\mathrm{pot}} \eta}\right)^{1 / 2} .
$$

Last, we define a root-mean-square stress

$$
\tau_{\mathrm{rms}} \equiv \eta \dot{\gamma}_{\mathrm{rms}}
$$

\section{RESULTS FOR CRYSTAL MUSHES}

\section{A. Viscosity under constant rotation rate}

Figures 4(a) and 4(b) show two typical profiles of torque and viscosity [deduced from Eq. (12)] as a function of time for $\omega=41.9 \mathrm{rad} \mathrm{s}^{-1}$ and $\phi_{\text {ice }}=0.42$ and $\phi_{\text {ice }}=0.51$.

In Fig. 4(a) the measured torque starts low, as the stirred pot contains only a sucrose solution at $+5^{\circ} \mathrm{C}$ and no ice. The increase in torque (and viscosity) up to $t \approx 5000$ s represents both the cooling of the solution and the buildup of ice volume fraction. After this, both the temperature and ice content have reached steady-state values, and it is the rheology from this time onwards that is the subject of the present paper.

Figure 4(b) shows the calculated $\eta(t)$ for ice mushes with $\phi_{\text {ice }}=0.42$ and 0.51 , as well as the viscosity of the sucrose solution serum phase at this temperature $(\phi=0)$ and the predicted value of the viscosity of a Krieger-Dougherty suspension in the same serum phase at volume fractions $\phi=0.42$ and 0.51 . In all cases, the mush viscosity is higher than the Krieger-Dougherty predictions for hard, nonattractive spheres.

The gradual decline of both torque and hence viscosity over the remainder of the experiment is due to the increase of crystal size through ripening. The decline of viscosity with time follows an approximate power law, while there is strong decrease of viscosity with increasing rotation rate $\omega$, shown in Fig. 5. Between runs at different ice volume fractions we see

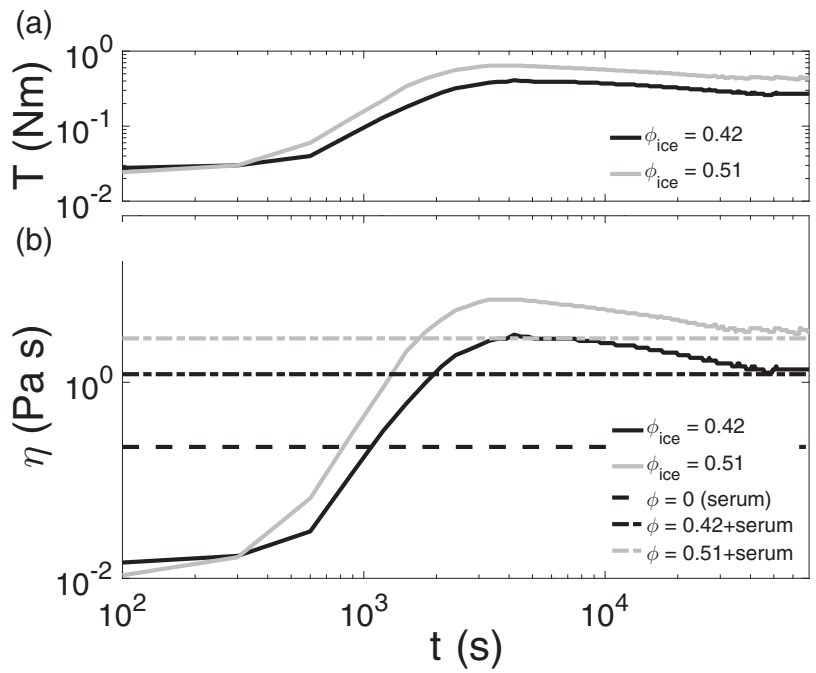

FIG. 4. (a) Variation in the torque profile as a function of time, for experiments where $\phi_{\text {ice }}=0.42$ and $\phi_{\text {ice }}=0.51$ at $\omega=41.9 \mathrm{rad} \mathrm{s}^{-1}$. (b) Profiles showing the change in viscosity over time as the solution crystallizes. The black curve shows the change in viscosity for $\phi_{\text {ice }}=0.42$, while the gray curve shows the data for $\phi_{\text {ice }}=0.51$, both stirred at $\omega=41.9 \mathrm{rad} \mathrm{s}^{-1}$. The dash-dotted lines show the expected viscosity for a sample with the same $\phi$ of hard spheres in equivalent serum. The dashed line shows the viscosity of the serum. The ice-sucrose suspension has a mean crystal size $R=100 \mu \mathrm{m}$ at $t=13200 \mathrm{~s}$. In these runs data were collected every $300 \mathrm{~s}$.

an increase in viscosity with increasing $\phi$; however, all runs reach peak $T$ (and hence $\eta$ ) at the same $t$. This is expected to be a result of clusters aggregating more efficiently under low shear conditions (due to the reduced force to break them up) and the increasingly tortuous routes the serum must flow

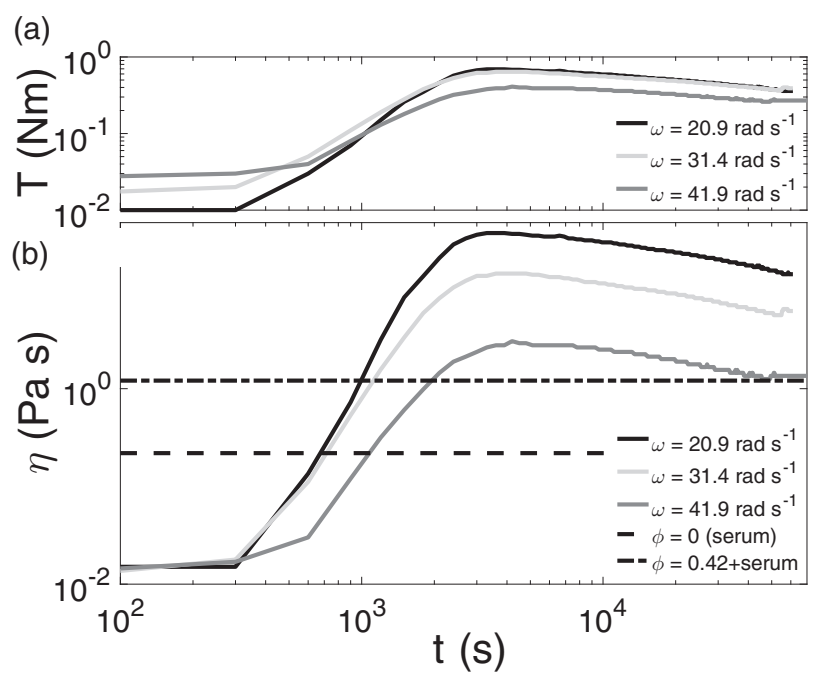

FIG. 5. (a) Profiles showing how torque varies through time for different values of $\omega$ for a suspension with $\phi_{\text {ice }}=0.42$. (b) Profiles showing how viscosity varies for the $\phi_{\text {ice }}=0.42$ samples with varied angular velocities. These experiments show the shear thinning nature of the ice-sucrose suspension. All shear rates show that the viscosity is greater than the suspension viscosity expected from Eq. (1). 


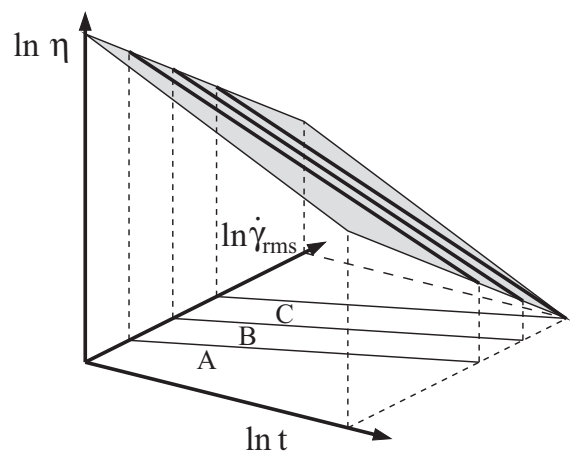

FIG. 6. Schematic of experimental procedure at constant rotation rate: Several experiments (A, B, and C in the figure) are performed at constant rotation rate over a period of time. This leads to changing $\dot{\gamma}_{\mathrm{rms}}$ over time $t$, and so the combined set of data points for time, viscosity $\eta$, and shear rate $\dot{\gamma}_{\text {rms }}$ (the latter two obtained from calibration) fall on a plane (up to experimental error) and are fitted to the power law of Eq. (4) using bivariate linear regression.

around the clusters. Shear thinning behavior is seen at higher $\omega$ as higher shear rates disrupt clusters, limiting the degree of aggregation that is possible and hence why high $\omega$ runs show viscosities closer to the Krieger-Dougherty prediction.

From these results, we observe that for the entirety of our run (postcrystallization peak) the viscosity is substantially larger than the Krieger-Dougherty result of noninteracting spheres (dash-dotted lines on Figs. 4 and 5). The viscosities decline with a power law, suggesting that the suspension viscosity may eventually drop to that of the Krieger-Dougherty value and level off. With decreasing $\omega$, the time this leveling off takes is increased.

Ultimately, we are interested in the exponents $n_{s}$ and $n_{r}$ in Eq. (2). However, in a given experiment at constant rotation rate, both $R$ and $\dot{\gamma}_{\text {rms }}$ are changing (the latter because the viscosity changes as the crystals grow in size). We therefore use Eq. (4) to obtain the combinations of exponents $n_{r} p_{t}$ and $n_{r} p_{s}+n_{s}$. Because both $t$ and $\dot{\gamma}_{\text {rms }}$ are changing, we need to perform a multivariate linear regression on runs at several different rotation rates to obtain the requisite combinations of exponents. The linear regression is shown schematically in Fig. 6, and the results are shown in Table II.

Since in these experiments, when conducted at different shear rates, the samples have undergone differing histories, we do not obtain a simple measurement of the shear thinning exponent $n_{s}$ alone. This can be seen in Eq. (4), where it is only the combination of exponents that is accessible. To remedy this, we look in the next subsection at experiments where a sample is sheared at a constant initial rate $\omega_{i}$, and then at a consistent time in the experiment the shear rate is stepped to a new value $\omega_{f}$.

\section{B. Viscosity after a step change in rotation rate}

In Sec. IV, for our calibration to be correct, our mushes are expected to see an instantaneously Newtonian response of the suspension to changes in shear rate. This is followed by a thixotropic relaxation period as the clusters reorganize and adapt to their new shear environment. A series of experiments have been run to observe this behavior. Samples are prepared in the same way as for the continuous experiments and placed in the stirred pot, set at $\omega_{i}=31.4 \mathrm{rad} \mathrm{s}^{-1}$ and $\Theta=-10^{\circ} \mathrm{C}$. Then, at $t=14400 \mathrm{~s} \omega$ is rapidly raised or lowered to a new rotation rate $\omega_{f}$. The suspension is then allowed to relax with no further changes in $\omega$. The impeller takes around $10 \mathrm{~s}$ to get up to the correct speed when the setpoint is changed and, as Fig. 7 shows, the relaxation time scale is considerably greater than $10 \mathrm{~s}$.

The results of the experiments show that although $T$ changes discontinuously, $\eta$ changes continuously (albeit inertia of the experimental apparatus is a potentially confounding effect). The viscosity displays a shear-thinning and thixotropic response [see Figs. 7 and 8(a)-8(d) for an increase in $\omega$ ], with a relaxation time scale $t_{\text {relax }} \approx 300$ s much greater than the time for a single rotation of the impeller. From analyzing the instantaneous $T, \eta$, and $\dot{\gamma}_{\text {rms }}$ at $t=14400 \mathrm{~s}$ and extrapolating to that time for the relaxed response, we can calculate $\eta$, $\dot{\gamma}_{\text {rms }}$ and $\tau_{\text {rms }}$ at the step point for both values of $\omega$. Plotting these data points in Figs. 9(a) and 9(b), we evaluate $n_{s}$ from Eq. (2) at $t=14400 \mathrm{~s}$. Since $R$ is constant, $n_{r}$ can be disregarded in this calculation and we find an average value of $n_{s}=-1.76 \pm 0.25$.

The uncertainty in $n_{s}$ is quite large, due to the scatter of the data and the limited range of rotation rates (and therefore $\dot{\gamma}_{\text {rms }}$ ) employed. The accessible shear rate range is limited by the current impeller geometry: Lower rotation rates lead to rapid clotting of the material and the formation of icy plugs, which give unusable datasets, while higher rotation rates can lead to air entrainment or spillage of material from the stirred pot. Despite this scatter, the data in Fig. 9 do not appear to support a relationship more complex than a single power law (which is also the result of our theoretical analysis). The effect of scatter and limited range of shear rates is that the true value of $n_{s}$ is

TABLE II. Experimental values for combinations of exponents [see Eqs. (3) and (4) in the text] for mushes under constant rotation rate $\omega$ (but varying $\dot{\gamma}_{\text {rms }}$ ). The results are obtained from multivariate linear regression, fitting either $\ln \eta$ or $\ln R$ to a linear function of $\ln t$ and $\ln \dot{\gamma}_{\text {rms }}$. Several experiments at differing but constant $\omega$ are used for each regression analysis. We observe in the last column a nonmonotonic behavior of our exponents with $\phi$. The standard deviations are from multiple experiments. Some quantities are not measured in particular experiments; these are denoted "n.m."

\begin{tabular}{lccccc}
\hline \hline$\phi$ (ice) & Values of $\omega / \mathrm{rad} \mathrm{s}^{-1}$ & $p_{t}$ & $p_{s}$ & $\left(n_{r} p_{t}\right)$ & $\left(n_{r} p_{s}+n_{s}\right)$ \\
\hline 0.42 & $20.9,31.4,41.9$ & $0.14 \pm 0.07$ & $-0.01 \pm 0.19$ & $-0.25 \pm 0.20$ & $-1.72 \pm 0.20$. \\
0.51 & $20.9,31.4,41.9$ & n.m. & n.m. & $-0.24 \pm 0.20$ & $-1.25 \pm 0.20$. \\
0.57 & $20.9,31.4$ & n.m. & n.m. & $-0.19 \pm 0.20$ & $-1.51 \pm 0.20$. \\
\hline \hline
\end{tabular}




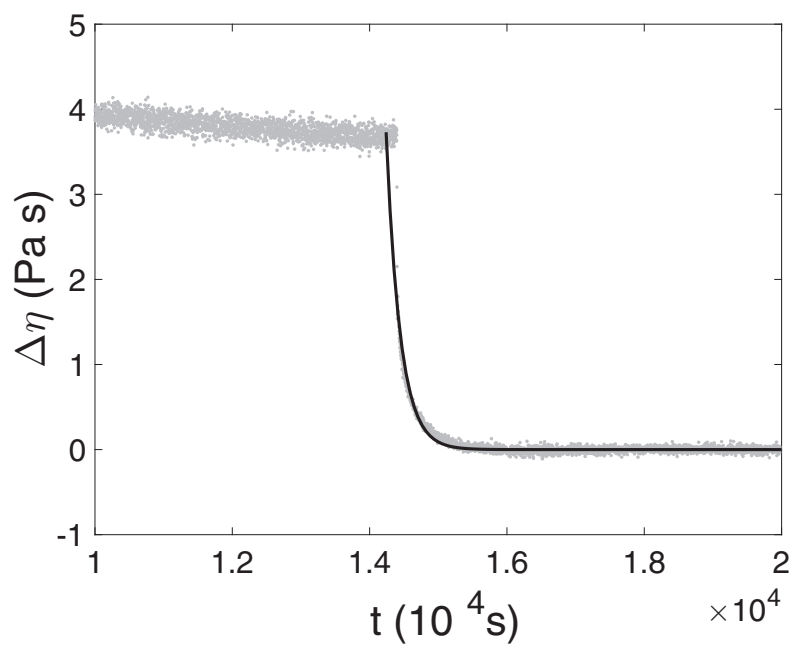

FIG. 7. Exponential decay of $\Delta \eta$ (gray points) as a function of time before and after a step change in rotation rate, where $\Delta \eta$ is the difference between the calculated viscosity and the relaxed viscosity at the new shear rate, extrapolated back to the time the rotation rate was changed. For this experiment $\phi_{\text {ice }}=0.42, \omega_{i}=31.4 \mathrm{rad} \mathrm{s}^{-1}$, and $\omega_{f}=41.9 \mathrm{rad} \mathrm{s}^{-1}$, with the step change in $\omega$ occurring at $t=14400 \mathrm{~s}$. The black dashed curve is the best fit exponential with the form $\Delta \eta=\Delta \eta_{0} \exp \left(-t / t_{\text {relax }}\right)$, and from this $t_{\text {relax }}$ can be determined.

likely to be slightly more negative than our estimate from the regression line in Fig. 9.

We can calculate the time scale of thixotropic relaxation $t_{\text {relax }}$ by fitting an exponential curve to the relaxation period where the long-term trend has been removed. This is shown in Fig. 7 and average values of $t_{\text {relax }}=370 \mathrm{~s}$ for both values of $\phi_{\text {ice }}$ are found in Table III.

(a)
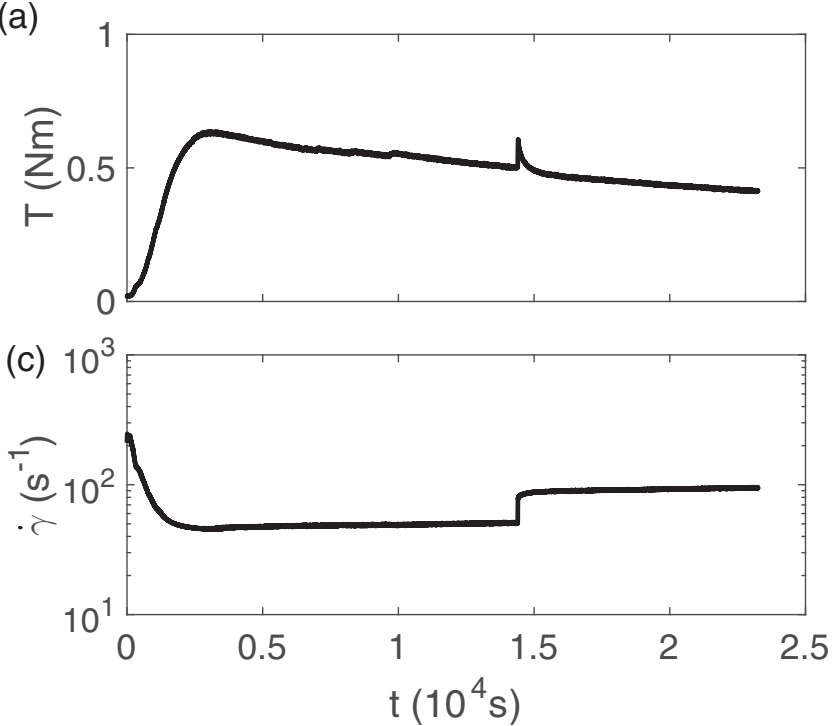

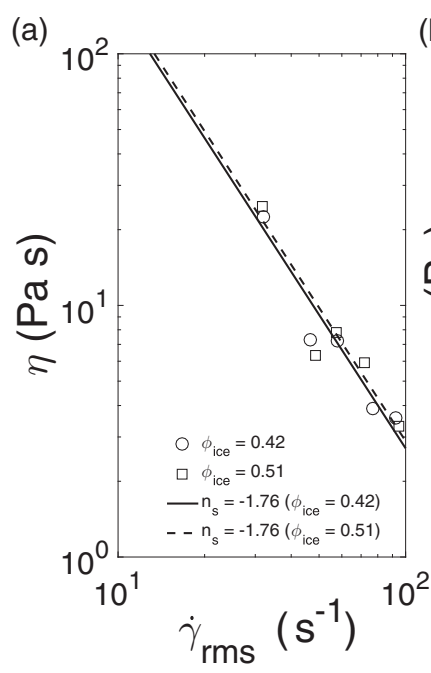

(b)

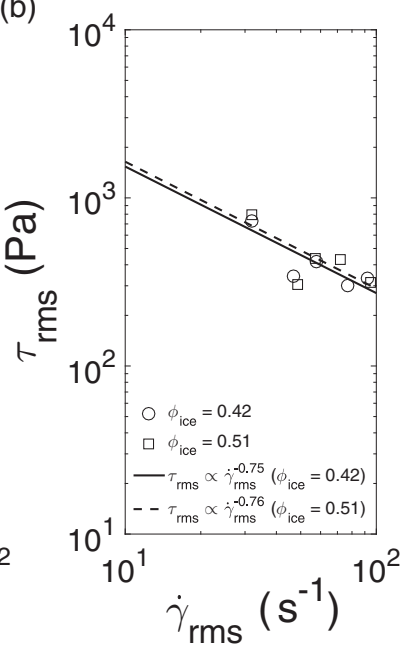

FIG. 9. (a) Plot showing the measured viscosity and root-meansquare shear rate at $t=14400 \mathrm{~s}$. The lines are best-fit lines, with prefactors related to $\phi$ and the exponents given as $n_{s}=-1.76$ \pm 0.23 for $\phi_{\text {ice }}=0.42$ and $n_{s}=-1.76 \pm 0.27$ for $\phi_{\text {ice }}=0.51$. (b) Plot showing the root-mean-square shear stress vs root-mean-square shear rate at $t=14400 \mathrm{~s}$. Both lines have a gradient of -0.75 . For both plots, error bars are the size of the markers.

\section{Crystal radius and shape}

The size and shape of the ice crystals produced were measured at various times during each run. The average radius $R$ of the ice crystals increases with time $t$, as seen in Figs. 10(a) and $10(\mathrm{~b})$.

At a fixed $T$, one might expect $R$ to increase as a power of time $t$ and also to depend on ice volume fraction and perhaps
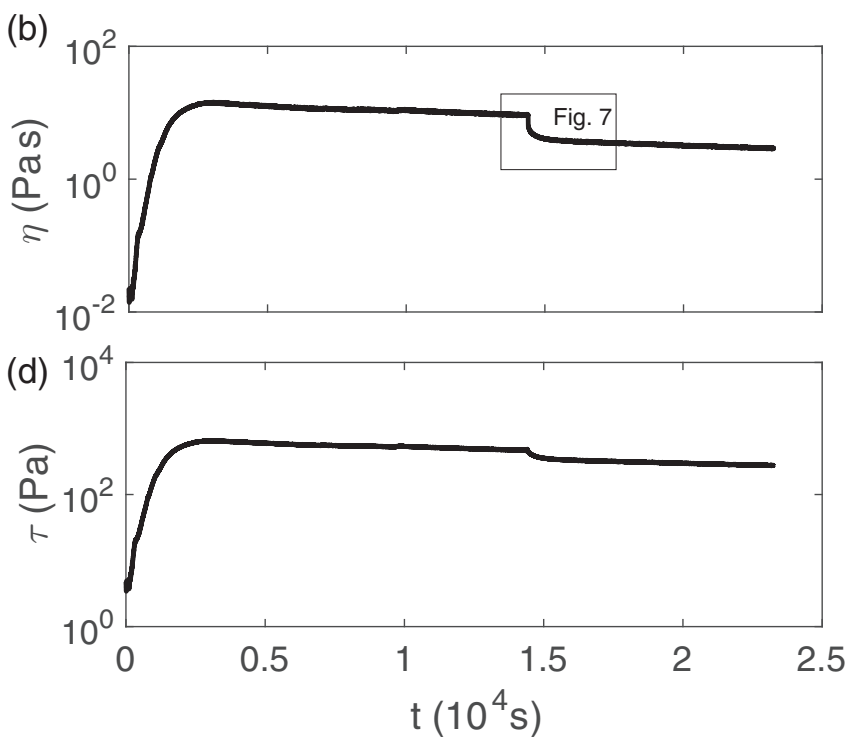

FIG. 8. Measured and calculated properties and how they evolve through a step change in $\omega$. For this experiment $\phi_{\text {ice }}=0.42, \omega_{i}=$ $31.4 \mathrm{rad} \mathrm{s}^{-1}$, and $\omega_{f}=41.9 \mathrm{rad} \mathrm{s}^{-1}$, with the step change at $t=14400 \mathrm{~s}$. (a) The measured torque changes discontinuously, with a sudden sharp increase when $\omega$ is dropped, before relaxing to below the original value. (b) $\eta$ [calculated from Eq. (12)] changes more continuously and shows a thixotropic relaxation following the change in $\omega$. Rectangle denotes portion which is shown in detail in Fig. 7. (c) $\dot{\gamma}_{\text {rms }}$ shows a jump before steadying. This value is calculated from Eq. (14). (d) Overall root-mean-square stress shows a small decrease due to the change in $\omega$, calculated from Eq. (15). In this experiment data are logged every $2 \mathrm{~s}$. 
TABLE III. Experimental values for the thixotropic viscosity relaxation time $t_{\text {relax }}$ calculated from the fitted exponential decay. Results are from experiments where the rotation rate starts at $\omega_{i}$, and at a time $t=14400 \mathrm{~s}$ is suddenly changed to a new value $\omega_{f}$. Errors are from the fitting of an exponential decay function to a single experimental run and do not include the additional scatter that would arise between replicates.

\begin{tabular}{lccc}
\hline \hline$\phi_{\text {ice }}$ & $\omega_{i} / \mathrm{rad} \mathrm{s}^{-1}$ & $\omega_{f} / \mathrm{rad} \mathrm{s}^{-1}$ & $t_{\text {relax }} / \mathrm{s}$ \\
\hline 0.42 & 31.4 & 20.9 & $204 \pm 6$ \\
0.42 & 31.4 & 26.2 & $354 \pm 5$ \\
0.42 & 31.4 & 36.7 & $554 \pm 9$ \\
0.42 & 31.4 & 41.9 & $622 \pm 13$ \\
0.51 & 31.4 & 20.9 & $400 \pm 10$ \\
0.51 & 31.4 & 26.2 & $391 \pm 7$ \\
0.51 & 31.4 & 36.7 & $182 \pm 8$ \\
0.51 & 31.4 & 41.9 & $243 \pm 13$ \\
\hline \hline
\end{tabular}

shear rate, as shown in Eq. (3). We believe that the secular increase in crystal size throughout an experiment at constant $\omega$ accounts for the slow decline in torque and (calculated) viscosity.

At zero shear rate, if crystals grow by Ostwald ripening [17], driven by the Laplace pressure of the ice-liquid interfacial energy $\sigma$ and limited by the interdiffusivity of solute and solvent through the liquid, then one would expect the ripening exponent $p_{t}=1 / 3$. This scaling has been observed not only in the dilute limit where Lifshitz-Slyozov-Wagner (LSW [18,19]) theory applies but also in more concentrated systems [44]. One would expect shear to accelerate the ripening by introducing mixing in addition to diffusion, but the only mechanism which would lead to a change of exponent $p_{t}$ is if interfacial attachment kinetics can limit ripening, rather than diffusion, resulting in a value of $p_{t}=1 / 2$ [19]. Mixing from shear might push a system into this regime from the diffusion-limited case.

However, from imaging the crystals in the mushes studied here, we observe for a range of conditions (see Fig. 11) that $R \propto t^{0.2}$ at fixed $\omega$, and depends only weakly on rotation rate. In order to obtain the exponents $p_{t}$ and $p_{s}$ in Eq. (3), we again note that at constant $\omega$, the shear rate will vary with time, so we need to perform bivariate regression analysis using a range of steady values of $\omega$ and fitting $\ln R$ to a linear combination of $\ln t$ and $\ln \dot{\gamma}_{\text {rmss }}$.

The results, shown in Table II, are that $p_{s}$ is small (indeed consistent with being zero), while $p_{t}$ takes the unexpectedly low value $p_{t}=0.14 \pm 0.07$. (Figure 11 shows a best-fit line with exponent $=0.2$ for all crystal sizes, but this includes some data that were excluded from the bivariate regression analysis since we only have different $\omega$ experiments for $\phi=$ 0.42.) A low value for the exponent $p_{t}$ (relative to theories of ripening) has also been seen previously in cryogenic ripening under shear [45], where ice has been observed crystallizing within a $\mathrm{NaCl}$ solution at different temperatures and shear rates. However, to our knowledge, no theory has predicted this exponent for ripening crystal systems.

As well as size, we measured the aspect ratios of crystals sampled from the stirred pot. The shape of the crystals does not change significantly with time, with the aspect ratio remaining

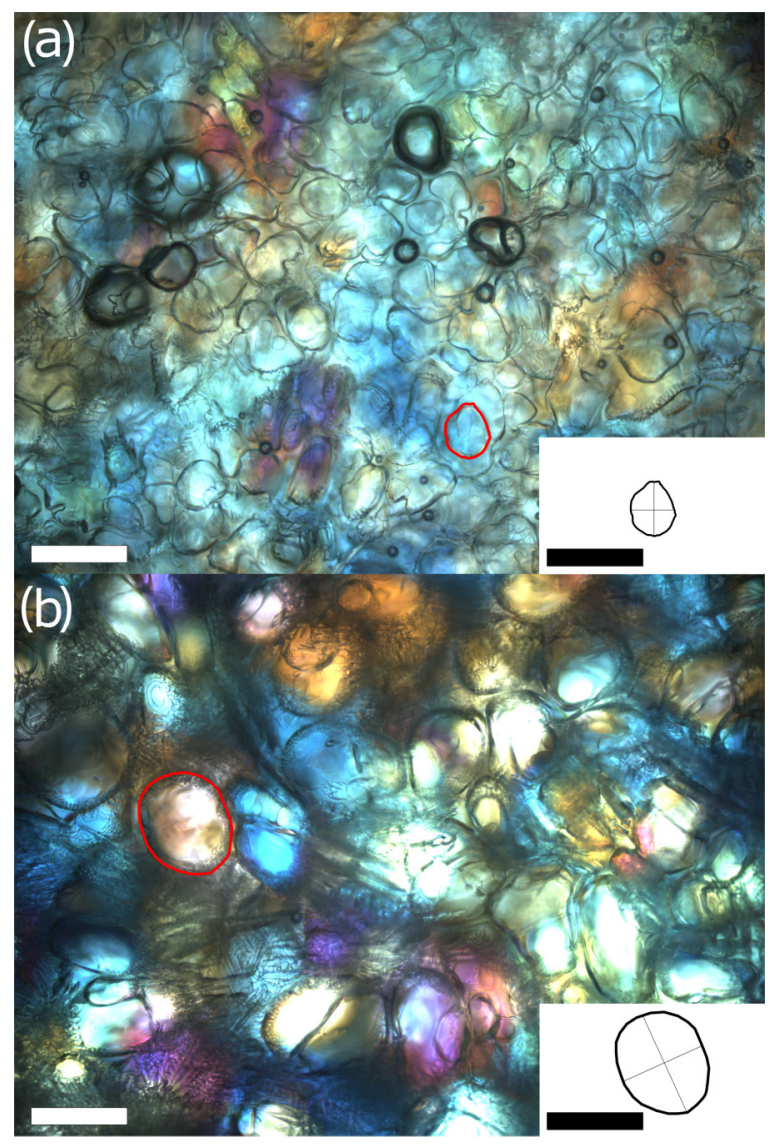

FIG. 10. Images of ice crystals after different residence times within the stirred pot, in a $\phi_{\text {ice }}=0.42$ suspension, with $\omega=$ $31.4 \mathrm{rad} \mathrm{s}^{-1}$. (a) After $t=4230 \mathrm{~s}$ (corresponding to the time of peak torque). (b) After $t=83000 \mathrm{~s}$. Scale bar for each is $200 \mu \mathrm{m}$. Insets are shown for one crystal, at the same scale, with the lines showing principal axes from which aspect ratio and long axis length are measured.

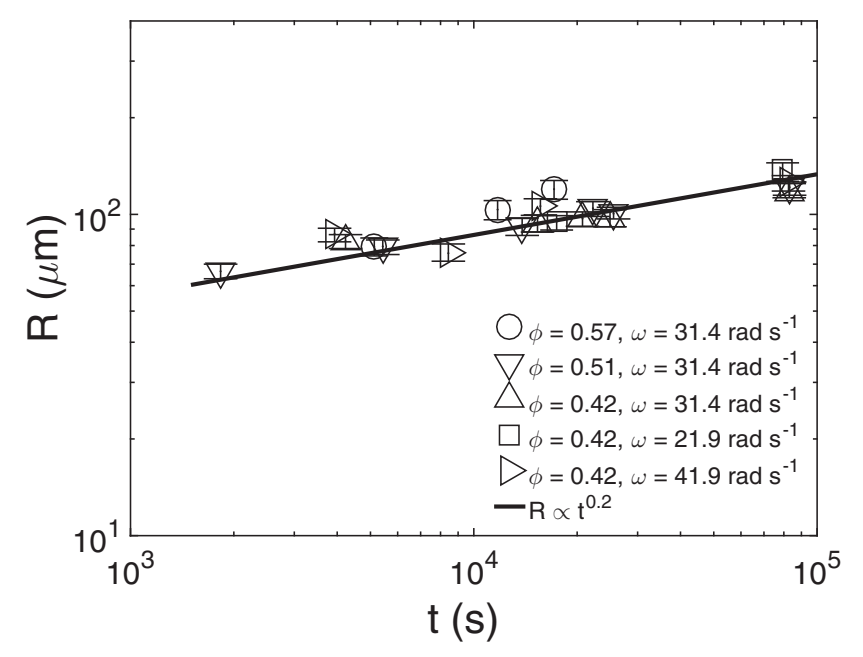

FIG. 11. Changes in crystal radius as a function of time in the stirred pot. Solid line is best-fit power law, with slope 0.2. Error bars show $95 \%$ confidence intervals, calculated using a bootstrap method. 


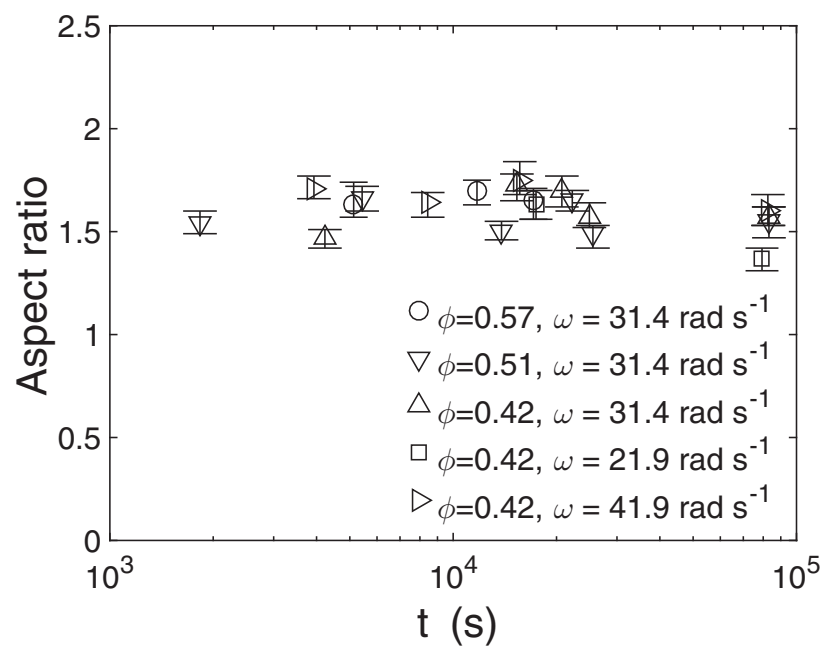

FIG. 12. Aspect ratio of ice crystals as a function of time in the stirred pot. Error bars show 95\% confidence intervals, calculated using a bootstrap method.

around 1.6, as shown in Fig. 12 (the same result as observed by Ref. [45]).

In some images of ice, however, the crystals appeared to be abraded, had rough surfaces, and were often misshapen (this can be seen in Fig. 13). Abrasion and breaking of partially sintered grains might be expected to occur under a high shear rate, so these processes are a possible cause for the unexpectedly small growth exponent $p_{t}$ we observed.

\section{Values and consistency of the exponents}

From the results in Tables II and Fig. 9(a), we deduce the four exponents that are predicted in Eqs. (2)-(4) using the step change experiments for $n_{s}$ and the constant $\omega$ experiments for $p_{t}, p_{s}$, and $n_{r} p_{t}$ to obtain

$$
\begin{gathered}
n_{s}=-1.76 \pm 0.25, \\
n_{r}=-1.8 \pm 1.3,
\end{gathered}
$$

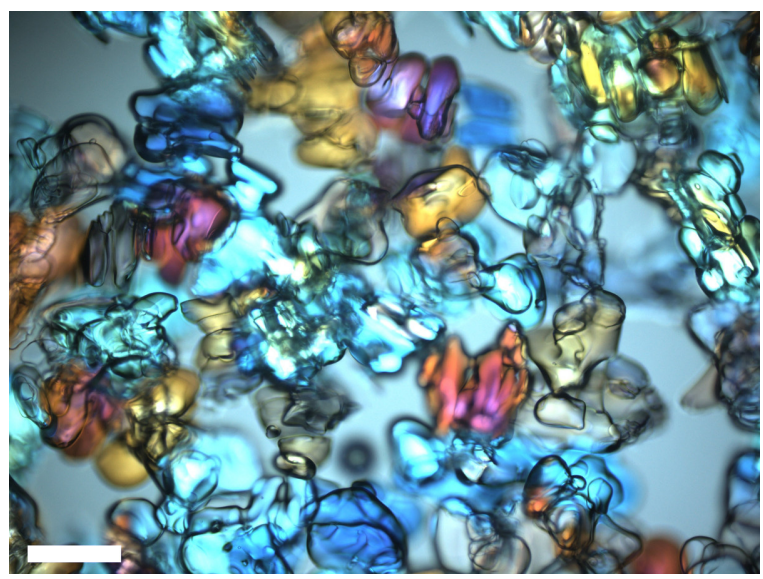

FIG. 13. Image of crystals in $\phi_{\text {ice }}=0.42$ at $\omega=31.4 \mathrm{rad} \mathrm{s}^{-1}$, at $t=25000 \mathrm{~s}$. There are numerous misshapen grains, with some showing instances of abrasion and damage. Scale bar is $200 \mu \mathrm{m}$.

$$
\begin{gathered}
p_{t}=0.14 \pm 0.07, \\
p_{s}=-0.01 \pm 0.19 .
\end{gathered}
$$

We also have an independent test of these exponents because we measure $n_{r} p_{s}+n_{s}$, but we have not used this in deriving Eqs. (16)-(19). We find, from these equations, $n_{r} p_{s}+n_{s}=$ $-1.78 \pm 0.34$. This is consistent with the measured value in Table II of $-1.49 \pm 0.12$.

\section{THEORETICAL MODELS}

\section{A. Stress arising from dynamic clusters}

In this section, we relate our observed rheology to the microstructural physics of the mushes.

At moderate to high volume fractions, the presence of even simple hard particles substantially increases the viscosity of a suspension over that of the serum. For our systems, the introduction of adhesive forces leads to a further large increase in viscosity, as seen in Fig. 5. There are two complementary ways to view this: through power dissipation and through force networks.

In the view based on power dissipation, for hard particles, this power is generated exclusively in the solvent. This remains essentially true if there are adhesive contacts, as long as they are brittle, so they break at very small strains. The increased viscosity arises from the tortuous paths and higher local shear rates imposed upon the serum as it flows around the particles. Even in the absence of adhesive forces, the flow must generate some correlations in particle position [46]; otherwise a simple self-consistent picture of particle pairs passing one another would predict a logarithmic divergence of viscosity with $\left(\phi-\phi_{m}\right)$, in contrast to the much stronger divergence predicted in Eq. (1). The high viscosities associated with adhesive forces must arise, in this picture, from the formation of extended structures (clusters) of crystals in the flow, which force even larger local strain rates on the serum phase.

The second picture is based on force networks. At high volume fractions, hydrodynamic forces become more localized between neighboring particles, so to a good approximation one can ignore the serum, replacing it with pairwise lubrication forces between the crystals.

The key assumption we make in this section is that the clusters formed in the flow are transient and in a quasisteady state (ignoring the slow ripening dynamics). That is to say, they form and break up over a time scale on the order of an inverse shear rate. More specifically, we suppose that any bond which forms between a pair of crystals survives only for a time $t_{\text {bond }} \sim \dot{\gamma}_{\text {rms }}^{-1}$ before being ruptured. This means that if there are clusters present, a typical tensional force between crystal pairs, in the extensional direction of the flow, is on the order of the rupture force $F_{\max }$ of the adhesive bond that is present. We assume that this sets the scale for all interparticle forces (extensional or compressive) in the flow.

Let the $i$ th component of the pair force between particles $m$ and $n$ be $F_{i}^{[m, n]}$, where the $j$ th component of the vector joining their centers is $r_{j}^{[m, n]}$, and let the representative volume of the suspension under consideration be $V$. The mean stress tensor 
in the suspension, with components $\tau_{i, j}$, will be given by [47]

$$
\tau_{i, j}=\frac{1}{2 V} \sum_{m, n} F_{i}^{[m, n]} r_{j}^{[m, n]} .
$$

Since contact forces are only possible between near neighbors, the number of pairs of particles per unit volume between which a nonzero force obtains will scale as $R^{-3}$ (with a prefactor that depends slightly on volume fraction near $\left.\phi_{m}\right)$. For moderate-to-high volume fractions, the length of each vector $\mathbf{r}^{[m, n]}$ will be close to $2 R$ and by assumption the forces are of typical magnitude $F_{\max }$, the rupture force of an adhesive bond. So from Eq. (20), we arrive at an estimate for the root-mean-square shear stress in the system:

$$
\tau_{\text {rms }} \propto F_{\max } R^{-2},
$$

where the constant of proportionality is dimensionless, of order unity, and depends only weakly on volume fraction near $\phi_{m}$.

We expect Eq. (21) to hold while there is a substantial amount of (transient) adhesive clusters in the flow. At sufficiently high shear rates, it is possible that breakup is so effective that all adhesive bonds are broken in a time much less than $\dot{\gamma}_{\text {rms }}^{-1}$, so there are effectively no clusters. As this state is approached, there will be a crossover to the hard particle (Krieger-Dougherty) viscosity.

The problem now reduces to finding the maximum force required to separate two crystals after they are brought into contact. We expect this to depend in a power-law manner on the crystal radius $R$ and the contact time $t_{\text {bond }} \sim \dot{\gamma}_{\text {rms }}^{-1}$. Substituting such a power law behavior into Eq. (21) and comparing to Eq. (2) allows us to relate the exponents (which will be predicted by the various theories that follow) to $n_{s}$ and $n_{r}$ :

$$
F_{\max } \propto \dot{\gamma}_{\mathrm{rms}}^{n_{s}+1} R^{n_{r}+2}
$$

In the following subsection, we present some theories from the literature for the bond strength $F_{\max }$ in terms of $t_{\text {bond }}$ and $R$.

\section{B. Theory for adhesive spheres}

Johnson-Kendall-Roberts (JKR) theory [48] describes the contact mechanics between two elastic spheres of radius $R$ when there is a reversible, adhesive interfacial energy per unit area $\sigma_{\text {surf }}$ between them if they touch. The resulting expression for the maximum force to separate them is

$$
F_{\text {max }}=\frac{3 \pi}{2} \sigma_{\text {surf }} R \text {. }
$$

From Eq. (22), this leads to predicted exponents $n_{s}=n_{r}=$ -1 .

Direct measurements of adhesive force have been performed on micromanipulated ice particles in air and sucrose solution [49]. These results are broadly consistent with JKR theory, but display a time dependence not present in the theory.

\section{Theory for sintering then brittle fracture}

Suppose two crystals have sintered together to form a neck of radius $x_{\text {sint }}$ with no elastic stresses present [Fig. 14(a)].
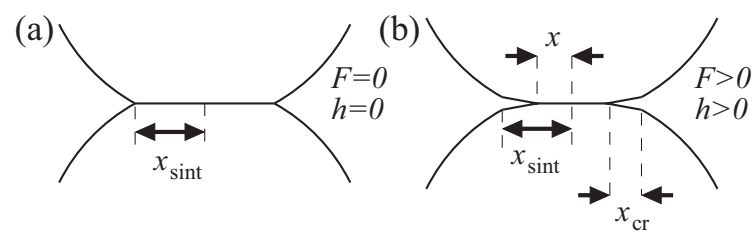

FIG. 14. (a) Geometry of two spheres of radius $R$ which have come into contact and formed a sintered neck, of radius $x_{\text {sint }}$. The applied tensional force $F$ is zero. (b) Geometry when a tensional force is applied. The sphere centers have moved apart a distance $h$ and the neck begins to fail, opening up an annular mode I crack of thickness $x_{\mathrm{cr}}$ so that the radius of the sintered contact disk reduces to $x=x_{\text {sint }}-x_{\mathrm{cr}}$.

Eventually, this neck will break by brittle fracture (mode I loading [50]) when a tensional force $F_{\max }$ is applied.

Consider the situation when a smaller force $F<F_{\max }$ is applied, under which the neck may open up an annular crack so that the new radius of the contact disk is $x<x_{\text {sint }}$ [see Fig. 14(b)]. The energy per unit area of the new surface created is $\sigma_{\mathrm{cr}}$, which for a brittle fracture will be similar to the ice-water or ice-vacuum surface energy, but for ductile fracture will be larger due to plastic deformation near the crack tip.

If we consider the scaling behavior, neglecting numerical factors of order unity, then the energy of the new surface created is

$$
U_{\text {surf }} \sim \sigma_{\text {cr }}\left(x_{\text {sint }}^{2}-x^{2}\right) .
$$

Under the action of the force, the center-to-center distance increases by $h$. The elastic deformation of the spheres is localized to a roughly isotropic region of radius $x$ around the center of symmetry of the pair [51]. Thus the deformed volume is of order $x^{3}$ and the strain of order $h / x$. The elastic energy stored is then

$$
U_{\mathrm{el}} \sim Y x h^{2},
$$

where $Y$ is the elastic modulus of the crystals.

The total energy of the system (surface, elastic, and the work done by the applied force) is thus

$$
U_{\text {tot }} \sim U_{\text {surf }}+U_{\text {el }}-F h .
$$

For an imposed force $F$ the system will choose $h$ and $x$ to minimize $U_{\text {tot }}$, under the constraint that $x$ cannot exceed $x_{\text {sint }}$ (and by definition $x \geqslant 0$ ).

Let us define some nondimensional parameters of the system:

$$
\begin{gathered}
\tilde{x} \equiv \frac{Y x}{\sigma_{\mathrm{cr}}}, \quad \tilde{h} \equiv \frac{Y h}{\sigma_{\mathrm{cr}}}, \quad \tilde{F} \equiv \frac{Y F}{\sigma_{\mathrm{cr}}^{2}}, \\
\Delta \tilde{U} \equiv \frac{Y^{2}\left(U_{\mathrm{tot}}-\sigma_{\mathrm{cr}} x_{\mathrm{sint}}^{2}\right) \sigma_{\mathrm{cr}}^{3}}{,}
\end{gathered}
$$

so that from Eq. (26) the nondimensionalized total energy is

$$
\Delta \tilde{U}=-\tilde{x}^{2}+\tilde{h}^{2} \tilde{x}-\tilde{F} \tilde{h} .
$$

Consider the behavior of the system when the force $F$ is imposed. The starting condition is $x=x_{\text {sint }}$ and $h=0$. The system will then follow a path downhill in $\Delta \tilde{U}$ in the space of $(\tilde{x}, \tilde{h})$. This means [see Fig. 15] that provided the separatrix of 

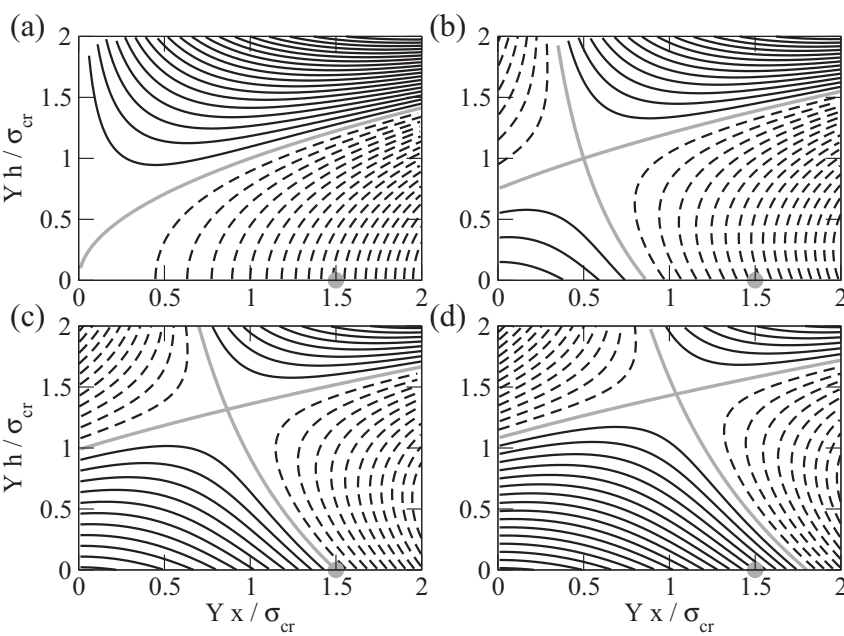

FIG. 15. Contour plots of the nondimensionalized total energy $\Delta \tilde{U}$ [see Eq. (29)] of two sintered spheres being pulled apart by a nondimensionalized force $\tilde{F}$. The initial conditions are shown by the gray circle and correspond in this example to $(\tilde{x}, \tilde{h})=(3 / 2,0)$. The contour through the saddle point is shown in gray and is the separatrix for two different behaviors of the system. Solid contours have values of $\Delta \tilde{U}$ greater than the saddle-point value, and dashed curves have values less than this. The system will follow a path of decreasing $\Delta \tilde{U}$, but is restricted to $\tilde{x} \leqslant 3 / 2$ at all times. (a) $\tilde{F}=0$. The initial condition is already the minimum energy point (under the constraint $\tilde{x} \leqslant 3 / 2$ ). (b) $\tilde{F}=1$. This is less than the critical value, and the minimum energy of the system is when $\tilde{x}$ takes its maximum value of $3 / 2$ and $\tilde{h}$ is greater than zero. (c) $\tilde{F}=3^{3 / 4}$, the critical value in this example. The separatrix passes through the initial conditions. (d) $\tilde{F}=3$, greater than the critical value. There is a path, always downhill in total energy, in which $\tilde{x} \rightarrow 0$ and $\tilde{h} \rightarrow \infty$, so the neck between the crystals breaks and they can separate to an arbitrarily large distance.

Eq. (29), which is the contour passing through the saddle point, lies to the left of the starting point, the minimum is achieved when $h$ is positive and $x=x_{\text {sint }}$. However, when $F$ increases to the point that the separatrix passes the initial condition [Fig. 15(d)], the system fails catastrophically and $h \rightarrow \infty$ and $x \rightarrow 0$, so the neck breaks and the crystals separate to an arbitrarily large distance.

The critical force for this to occur is $F_{\max }$, which can be found as follows: Treating Eq. (29) as quantitative, the value of $\Delta \tilde{U}$ at the saddle point, where $\partial \Delta \tilde{U} / \partial \tilde{x}=\partial \Delta \tilde{U} / \partial \tilde{h}=0$ is $-(3 / 4) \tilde{F}^{4 / 3}$. The equation for the separatrix is therefore

$$
-(3 / 4) \tilde{F}^{4 / 3}=-\tilde{x}^{2}+\tilde{h}^{2} \tilde{x}-\tilde{F} \tilde{h},
$$

and this curve intersects the $\tilde{x}$ axis at $\tilde{x}=(\sqrt{3} / 2) \tilde{F}^{2 / 3}$. The critical force occurs when this intersection coincides with the initial condition $\tilde{x}=Y x_{\mathrm{sint}} / \sigma_{\mathrm{cr}}$, so restoring dimensions using Eq. (27) we find (up to an unknown numerical prefactor) that

$$
F_{\max } \propto\left(Y \sigma_{\text {cr }} x_{\text {sint }}^{3}\right)^{1 / 2} .
$$

In order to complete this model, we need to know how the radius $x_{\text {sint }}$ of the sintered junction between two crystals grows with contact time. Although various mechanisms of sintering are possible [16,42], including plastic flow, van der Waals attraction, and vacancy diffusion in the crystal, it is likely that bulk-diffusion-limited liquid-phase sintering is the dominant process for a pair of molecularly rough [52] crystals brought into contact when immersed in a solution of their melt. Even for this process, various theories have been put forward in the literature to describe the growth of the neck radius $x_{\text {sint }}$ with contact time $t_{\text {bond }}$ (where, to reiterate, in the flow, we will choose $t_{\text {bond }}=\dot{\gamma}_{\mathrm{rms}}^{-1}$ ).

For liquid phase sintering, consider the surface mean curvature $\kappa$ near the neck region of the pair of sintering crystals. Assuming a crystal has a roughly isotropic surface energy $\sigma_{\text {surf }}$ and a latent heat of fusion $L_{f}$ per unit volume, the Gibbs-Thomson effect [53] states that if a flat crystal surface has an (absolute) melting temperature $\Theta_{m}$, the melting point of a curved crystal surface will be changed by an amount

$$
\delta \Theta_{m}=\Theta_{m} \sigma_{\text {surf }} \kappa / L_{f} .
$$

For our systems, the crystals are not in contact with their pure melt, but with a solution of sucrose, so that at some temperature $\Theta$, there will be a mass fraction $c_{\text {eq }}(\Theta)$ of solute that is in equilibrium with a flat crystal surface. This dissolution curve must be found empirically. Assuming Eq. (32) applies to the dissolution curve when there is solute present, a simple graphical construction shows that at constant temperature, curvature induces a change $\delta c$ in the equilibrium solute mass fraction in contact with ice [54]:

$$
\delta c \approx-\Theta \frac{d c_{\mathrm{eq}}}{d \Theta} \frac{\kappa \sigma_{\mathrm{surf}}}{L_{f}} .
$$

For water-sucrose systems, thermal diffusion is much faster than mass diffusion [55], so the system remains isothermal. Surface curvature induces changes in water concentration in the serum phase, and neck growth is driven by mass diffusion down the resulting concentration gradients.

A naïve theory of sintering by dissolution and precipitation posits, on geometrical grounds, that

$$
\kappa \approx \frac{R}{x_{\text {sint }}^{2}},
$$

and furthermore that the concentration difference of Eq. (33) operates over a length scale of order $R$. Therefore, the prediction would be that $d x_{\text {sint }} / d t_{\text {bond }} \approx D \delta c / R$, where $D$ is the interdiffusivity of solute and solvent in the unfrozen serum phase. This can be rearranged to give

$$
\frac{x_{\text {sint }}}{R} \approx\left(\frac{D \Theta \sigma_{\text {surf }}}{R^{3} L_{f}}\left|\frac{d c}{d \Theta}\right| t_{\text {bond }}\right)^{\beta},
$$

where $\beta=1 / 3$. Substituting Eq. (35) into Eq. (31) and then Eq. (22) leads to the predicted exponents

$$
\begin{aligned}
& n_{s}=-1-\frac{3 \beta}{2}, \\
& n_{r}=-\frac{1}{2}-\frac{9 \beta}{2} .
\end{aligned}
$$

For $\beta=1 / 3$, this leads to predictions of $n_{s}=-3 / 2$ and $n_{r}=$ -2 .

In contrast, Courtney [56] argued that diffusion in the narrowing (wedge-shaped) gap between the two spheres is likely to be significantly hindered by the geometry, so that by considering diffusive trajectories and the mass of material that 


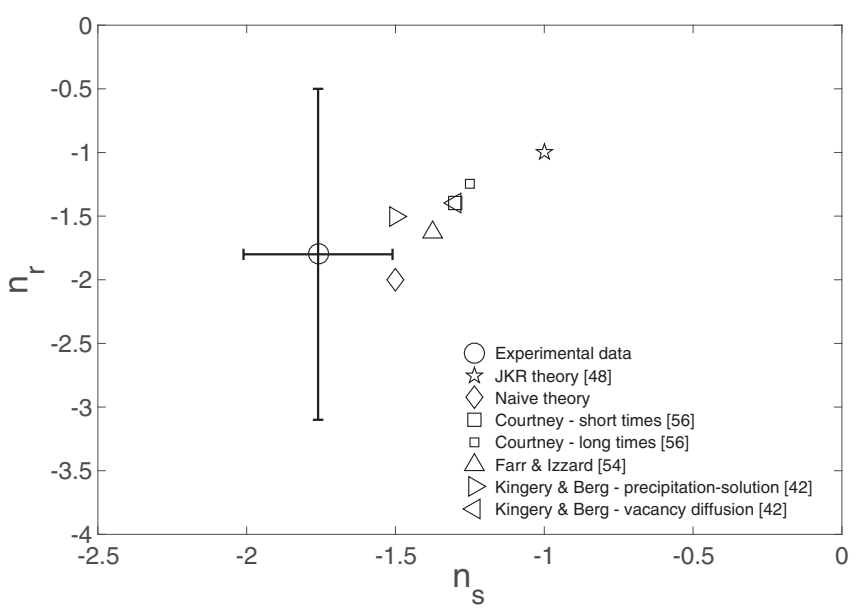

FIG. 16. Plot showing how the experimentally derived values of $n_{r}$ and $n_{s}$ compare to the theoretical values given in Table IV.

gets added to the neck, it is possible to conclude that the form of Eq. (35) is correct, but with $\beta=1 / 5$ or $\beta=1 / 6$ (depending on detailed assumptions of the time scales involved). Another recent theory from Farr and Izzard [54] points out that Eq. (34) is unlikely to be correct, as the narrowest region of the neck may become significantly blunted. By considering a teardrop-shaped solitary wave solution for the sintering of two parallel sheets, the authors ultimately arrive at a prediction again of the form of Eq. (35), but with $\beta=1 / 4$.

Kingery and Berg [42] provide scalings for $x_{\text {sint }}$ for several different sintering processes. For evaporation-condensation they find

$$
x_{\text {sint }} \propto R^{1 / 3} t_{\text {bond }}^{1 / 3},
$$

while for diffusion in the crystal

$$
x_{\text {sint }} \propto R^{2 / 5} t_{\text {bond }}^{1 / 5} \text {. }
$$

The predicted exponents for all of these mechanisms are shown in Table IV and are compared to the exponents collected from the experimental analysis (Sec. VD) in Fig. 16. The models of JKR [48] and Courtney [56] can be ruled out as they lie well outside the experimental uncertainties. We believe that the vacancy-diffusion model of Kingery and Berg [42] should also be eliminated, as self-diffusivity in ice at this temperature (around $1.3 \times 10^{-15} \mathrm{~m}^{2} \mathrm{~s}^{-1}$ [57]) is much lower than the diffusivity of water in the serum phase (and further the vacancy or interstitial fraction is low in ice crystals). We do see agreement, within experimental uncertainties, between our experimental dataset and the exponents found from the naïve liquid phase sintering theory and the evaporation-condensation model of Kingery and Berg [42].

The large error bars on the values of the rheological exponents are mainly due to limitations on the size of the shear rate range collected (as discussed above) and could perhaps be reduced with different experimental apparatus. It is also notable that there is dispute in the literature about the theoretical exponents for neck growth, even when considering the single mechanism of sintering by diffusion through the liquid phase. It would be desirable to obtain direct experimental data, from optical microscopy, on the dynamics of neck growth between crystals. However, this is experimentally challenging, as neck growth happens on a time scale of order a reciprocal shear rate (longer than the time for sample collection and transport to the microscope) and it is not clear that the resolution of the current apparatus would be sufficient. More theoretical or simulation work in this area would therefore be desirable.

\section{CONCLUSIONS AND IMPLICATIONS}

We have found that the viscosity of ice-sucrose mushes has a power-law dependence on shear rate and crystal size. These powers can be explained due to the formation of clusters of crystals by liquid-phase sintering and breakup of these clusters by brittle fracture under flow. We have observed under long time scales the crystals ripen under shear, with a power of time that is smaller than any published theory. We interpret this as possibly due to abrasion or effects of cluster breakup on the crystals.

We believe that the present work, on a model system with subspherical, sintering crystals, represents a step towards understanding the rheology of a much broader class of materials which are of great technological and social importance. This class of material includes volcanic and cryogenic lavas, frazil ice, frozen foods, and salt slurries.

The phenomenology of ice-sucrose systems should be directly relevant to the behavior of cryogenic lavas, which have been discovered widely throughout the moons and minor planets beyond Mars, for example, Ceres [7], Ganymede [58], Titan, Enceladus [6], Triton [59], and Pluto [60]. These lavas have various ices present as crystal phases; therefore, one would expect liquid-phase sintering as suggested here will

TABLE IV. Theoretical predictions for the exponents $n_{s}$ and $n_{r}$ in Eq. (2) from different literature sources, as analyzed in Sec. VI, compared to the experimental exponents from this study.

\begin{tabular}{lcc}
\hline \hline Theoretical model & $n_{s}$ & $n_{r}$ \\
\hline JKR adhesive spheres [48] & -1 & -1 \\
Naïve theory of fracture and liquid-phase sintering & $-3 / 2$ & -2 \\
Fracture and liquid-phase sintering by diffusion, short times (Courtney [56]) & $-13 / 10$ & $-7 / 5$ \\
Fracture and liquid-phase sintering by diffusion, long times (Courtney [56]) & $-5 / 4$ & $-5 / 4$ \\
Fracture and liquid-phase sintering (Farr and Izzard [54]) & $-11 / 8$ & $-13 / 8$ \\
Fracture and sintering by evaporation-condensation (Kingery and Berg [42]) & $-3 / 2$ & $-3 / 2$ \\
Fracture and sintering by vacancy diffusion (Kingery and Berg [42]) & $-13 / 10$ & $-7 / 5$ \\
Experimental results & $-1.76 \pm 0.25$ & $-1.80 \pm 1.30$ \\
\hline \hline
\end{tabular}


be relevant to the features produced by such flows [5]. These results will be of more limited importance to the study of terrestrial lavas, as in general the rheology of such flows can be described by a modified Krieger-Dougherty relationship [3].

For large bodies of magma which contain reservoirs of crystal mush, the situation is different. Liquid-phase sintering has been predicted to occur in granitic mushes with a high degree of partial melt and confirmed through dihedral angles between quartz-feldspar and quartz-quartz grain junctions [61]. There is also extensive evidence of solution-precipitation sintering dynamics in olivine-basalt aggregates [62,63].

Knowing viscosity is of crucial importance in understanding the behavior of magmatic hazards. Crystal-rich ignimbrites (from highly voluminous, explosive eruptions) are observed in the rock record [64], with the crystals expected to come from a long-lived stored mush reservoir [65], which has been triggered due to a change in the thermal state of the reservoir [64]. Although the Krieger-Dougherty relationship is often used in the literature to describe eruptible magmas with $\phi \approx 0.5-0.6$ [64], the yield stress behavior just noted and the shear rate and time dependence that we have uncovered in the present work suggest that eruptibility of these magmas is being overstated (and the viscosity vastly underestimated) by the Krieger-Dougherty relation.

Moving beyond subspherical crystals, frazil ice forms due to turbulent mixing of supercooled salt water, often in polynyas near ice shelves [66]. The ice crystals formed are needle shaped and often stick together in clots. We believe the rheology of mushes of high-aspect-ratio crystals is both of critical importance (since it applies to many magmatic systems as well as frazil ice) but has received little study in the literature. Such systems bring added complexity and experimental challenges from the possibility for flow-induced alignment.

\section{ACKNOWLEDGMENTS}

The authors gratefully acknowledge William Frith, Jerome Neufeld, and Marian Holness for many useful discussions. A.J.G. acknowledges a National Environment research council collaborative awards in science and engineering (NERC CASE) studentship award [Grant No. D07591442: MA-2013$00657]$.
[1] H. R. Shaw, J. Petrol. 10, 510 (1969).

[2] L. Caricci et al., Earth Planet. Sci. Lett. 264, 402 (2007).

[3] A. Vona et al., Geochim. Cosmochim. Acta 75, 3214 (2011).

[4] L. H. Smedsrud and A. Jenkins, J. Geophys. Res., Oceans 109, C03025 (2004).

[5] R. M. C. Lopes et al., Icarus 186, 395 (2007).

[6] F. Zhong et al., Icarus 202, 607 (2009).

[7] O. Ruesch et al., Science 353, 4286 (2016).

[8] C. Clark, The Science of Ice Cream (RSC Publishing, Cambridge, 2004).

[9] J. R. Stokes, J. H. Telford, and A-M. Williamson, J. Rheol. 49, 139 (2005).

[10] I. Dinçer and M. Kanoǵlu, Refrigeration Systems and Applications (Wiley, New York, 2010).

[11] A. K. Dahle and D. H. St. John, Acta Mater. 47, 31 (1998).

[12] J. T. Black and R. A. Kohser, DeGarmo's Materials and Processes in Manufacturing (Wiley, New York, 2011).

[13] W. B. Sudweeks, Ind. Eng. Chem. Prod. Res. Dev. 24, 432 (1985).

[14] C. A. Baar, Applied Salt-Rock Mechanics 1: The In-Situ Behavior of Salt Rocks (Elsevier, Amsterdam, 2013).

[15] H. C. Hamaker, Phys. (Amsterdam, Neth.) 4, 1058 (1937).

[16] R. M. German, P. Suri, and S. J. Park, J. Mater. Sci. 44, 1 (2009).

[17] W. Ostwald, Z. Phys. Chem. 22, 289 (1897).

[18] I. M. Lifshitz and V. V. Sloyozov, J. Phys. Chem. Solids 19, 35 (1961).

[19] C. Z. Wagner, Z. Elektrochem. 65, 581 (1961).

[20] J. S. Kargel et al., Icarus 89, 93 (1991).

[21] J. Mewis and N. J. Wagner, Adv. Colloid Interface Sci. 147, 214 (2009).

[22] A. Einstein, Ann. Phys. 324, 289 (1906).

[23] G. B. Jeffery, Proc. R. Soc. London, Ser. A 102, 161 (1922).

[24] C. G. de Kruif et al., J. Chem. Phys. 83, 4717 (1985).

[25] J. Bernal and J. Mason, Nature (London) 188, 910 (1960).
[26] C. Song, P. Wang, and H. A. Makse, Nature (London) 453, 629 (2008).

[27] H. M. Jaeger, S. R. Nagel, and R. P. Behringer, Rev. Mod. Phys. 68, 1259 (1996).

[28] R. S. Farr, J. R. Melrose, and R. C. Ball, Phys. Rev. E 55, 7203 (1997).

[29] I. M. Krieger and T. J. Dougherty, Trans. Soc. Rheol. 3, 137 (1959).

[30] G. W. Delaney and P. W. Cleary, Europhys. Lett. 89, 34002 (2010).

[31] G. W. Delaney, J. E. Hilton, and P. W. Cleary, Phys. Rev. E 83, 051305 (2011).

[32] R. S. Farr and R. D. Groot, J. Chem. Phys. 131, 244104 (2009).

[33] R. S. Farr, J. Chem. Phys. 141, 214503 (2014).

[34] T. A. Witten and L. M. Sander, Phys. Rev. Lett. 47, 1400 (1981).

[35] M. Y. Lin et al., Nature (London) 339, 360 (1989).

[36] R. Buscall et al., J. Chem. Soc., Faraday Trans. 1 84, 4249 (1988).

[37] R. Buscall, P. J. Scales, A. D. Stickland, H-E. Teo, and D. R. Lester, J. Non-Newtonian Fluid Mech. 221, 40 (2015).

[38] R. Buscall et al., J. Non-Newtonian Fluid Mech. 222, 112 (2015).

[39] P. Schall and M. van Hecke, Annu. Rev. Fluid Mech. 42, 67 (2010).

[40] H. A. Barnes, An Introduction to Rheology (Elsevier, Amsterdam, 1989).

[41] M. Wyart and M. E. Cates, Phys. Rev. Lett. 112, 098302 (2014).

[42] W. D. Kingery and M. Berg, J. Appl. Phys. 26, 1205 (1955).

[43] F. M. Beckett, H. M. Mader, J. C. Phillips, A. C. Rust, and F. Witham, J. Fluid Mech. 682, 652 (2011).

[44] L. Bremer et al., Part. Part. Syst. Charact. 13, 350 (1996).

[45] P. Pronk et al., Int. J. Refrig. 28, 27 (2005).

[46] G. Marrucci and M. M. Denn, Rheol. Acta 24, 317 (1985). 
[47] M. P. Allen and D. J. Tildesley, Computer Simulation of Liquids (Clarendon Press, Oxford, UK, 1987).

[48] K. L. Johnson, K. Kendall, and A. D. Roberts, Proc. R. Soc. London, Ser. A 324, 301 (1971).

[49] X. Fan, et al., Powder Technol. 131, 105 (2003).

[50] A. T. Zehnder, Fracture Mechanics (Springer, Berlin, 2012).

[51] H. R. Hertz, J. Angew. Mathematik 92, 156 (1882).

[52] W. K. Burton, N. Cabrera, and F. C. Frank, Philos. Trans. R. Soc. London, Ser. A 243, 299 (1951).

[53] F. Meissner, Z. Anorg. Allg. Chem. 110, 169 (1920).

[54] R. S. Farr and M. J. Izzard, Phys. Rev. E 77, 041608 (2008).

[55] W. M. Haynes, CRC Handbook of Chemistry and Physics (CRC Press, Boca Raton, FL, 2016).

[56] T. H. Courtney, Metall. Trans. A 8, 671 (1977).
[57] B. Geil, T. M. Kirschgen, and F. Fujara, Phys. Rev. B 72, 014304 (2005).

[58] A. P. Showman, I. Mosqueira, and J. W. Head III, Icarus 172, 625 (2004).

[59] A. D. Fortes et al., Icarus 188, 139 (2007).

[60] J. M. Moore et al., Science 351, 1284 (2016).

[61] S. R. Jurewicz and E. B. Watson, Geochim. Cosmochim. Acta 49, 1109 (1985).

[62] R. F. Cooper and D. L. Kohlstedt, Phys. Chem. Miner. 11, 5 (1984).

[63] R. F. Cooper and D. L. Kohlstedt, J. Geophys. Res. 91, 9315 (1986).

[64] C. Huber, O. Bachmann, and J. Dufek, Geology 40, 115 (2012). [65] K. M. Cooper and J. R. Kent, Nature (London) 506, 480 (2014). [66] S. Martin, Annu. Rev. Fluid Mech. 13, 379 (1981). 